\title{
The Development of a Quantitative RCM/MSG Application using Microsoft Office
}

\author{
R Sianturi ${ }^{1}$, E Suwondo ${ }^{1, *}$ \\ 1 Faculty of Mechanical and Aerospace Engineering, Institute of Technology Bandung, Jl. Ganesha 10 \\ Bandung 40132, Indonesia \\ * Correspondence: esuwondo@ae.itb.ac.id
}

Received: 18 July 2020; Accepted: 15 November 2020; Published: 20 December 2020

\begin{abstract}
The number of small to medium scale industries makes the development of a low cost and easy to use application to support maintenance programs development is necessary. Besides for industries, the developed application can be used also in the academic field for research. For cost reasons, the application is developed using Microsoft Access and Microsoft Excel. The development of the application is carried out in three steps, i.e. the determination of application specifications, the programming in Microsoft Access and Microsoft Excel, and the validation and user-friendly test. The application is developed by considering the relationship between the risk quantification of a failure effect and the selection of a maintenance task and its interval. The effectiveness assessments are based on the failure risk reduction up to an acceptable level. The developed application is named "Quantitative RCM/MSG". The application can conduct a database management according to the steps of the RCM/MSG method. The application can determine the required maintenance tasks and intervals for the category of evident and hidden failure effects by taking the safety and financial aspects into account. This application does its functions correctly and easy to use. For further application development, application can be developed using series and combination systems. Application with module redesign on default action.
\end{abstract}

Keywords: support maintenance programs, risk quantitative, failure effects, Microsoft Office

\section{Introduction}

Doing maintenance to a company's product is very important. Maintenance itself can affect to safety, maintenance costs, the reliability of system and productivity. Reliability-Centered Maintenance (RCM) or Maintenance Steering Group-3 (MSG-3) is one of the process used to make maintenance programs. Reliability-Centered Maintenance (RCM) or Maintenance Steering Group-3 (MSG-3) is one of the processes used to make maintenance programs. RCM is a method used to determine what must be done to ensure each physical asset to keep working according to what is desired or in accordance with the process to determine effective care [1]. MSG-3 is a method used to determine maintenance tasks and scheduled maintenance intervals to avoid large losses and ensure physical assets continue to work as desired [2].

RCM / MSG-3 is a process used to create maintenance programs. In the preparation of the treatment program will be selected quantitative care assignments for each failure that occurs. Failure quantification is done to be able to assess the effects of failure that occur quantitatively. The value of the failure effect will be used to determine the selection of maintenance tasks by paying attention to their effects on safety and financial failure. 
It takes an inexpensive and easy to use application with users who don't have to be experienced or are still beginners. Current technological developments make it possible to develop an application in the selection of maintenance tasks. Microsoft Access is used in developing an application for determining maintenance tasks based on the RCM / MSG-3 process, especially in the quantification of failures and selection of maintenance tasks.

\section{Materials and Methods}

\subsection{Reliability-Centered Maintenance (RCM)}

Reliability Centered Maintenance (RCM) is a process used to determine what must be done to ensure that each physical asset works as desired $[1,3]$. There are steps in the RCM process, namely:

1. Identify the function and standard of achievement of items or components

2. Identify failure conditions

3. Identify failure mode

4. Identify the effects of failure

5. Identify the consequences of failure

6. Maintenance task selection

7. Determining default actions

\subsection{Maintenance Steering Group-3 (MSG-3)}

MSG-3 is a method developed by ATA (Air Transport Association of America) which aims as a means to determine maintenance tasks and scheduled maintenance intervals. The main objective of MSG-3 is to assist in determining and establishing scheduled care tasks and treatment intervals. Types of care tasks based on MSG-3:

a. Lubrication/Servicing (LU/SV)

b. Operational/Visual check (OP/VC)

c. Inspection/Functional check (IN/FC)

d. Restoration/Overhaul (RS)

e. Discard (DS)

\subsection{Three Parameters of Weibull Distribution}

Weibull distribution is a method used to estimate the probability of failure of an item or component. Three parameters of Weibull distribution are shape $(\beta)$ parameter, scale $(\eta)$ parameter, and location $(\gamma)$ parameter. Three parameters Weibull equation is a probability of density function which is formulated as follows.

$$
f(T)=\frac{\beta}{\eta}\left(\frac{T-\gamma}{\eta}\right)^{\beta-1} \cdot e^{-\left(\frac{T-\gamma}{\eta}\right)^{\beta}}
$$

Shape $(\beta)$ parameter Weibull distribution is also known as slope or line gradient from the plot of the probability of density function (pdf). By knowing the shape paramete, the scale parameter can be known using the following equation:

$$
\eta=e^{-\left(\frac{\text { intercepts }}{\text { slope }}\right)}
$$


For location parameters, the method developed by Muralidhar, Huamurty and Zanakis is used with the following equation [4].

$$
\gamma=\frac{T_{1} T_{n}-T_{j}^{2}}{T_{1}+T_{n}-2 T_{j}}
$$

$T 1$ : First data

$T n$ : The last value of the $\mathrm{n}$ sample

$T j$ : j value

$\boldsymbol{j}_{\text {uncensored }}=\boldsymbol{n} \boldsymbol{p}$ (rounded up to get the data)

$\boldsymbol{j}_{\text {censored }}=\boldsymbol{n} \boldsymbol{p}\left(\frac{n}{N}\right) \quad$ dan $\quad \boldsymbol{p}=\mathbf{0 . 8 8 2 9} \boldsymbol{n}^{-0.3437}$

Based on the three parameters of the Weibull distribution, can be determined the value of the unreliability of item. Unreliability is the probability of failure of a unit in completing a mission at a specified time with certain operating conditions denoted by $\mathrm{F}(\mathrm{T})$. The equation for determining the unreliability value is as follows:

$$
\begin{aligned}
& \boldsymbol{R}(\boldsymbol{T})=e^{-\left(\frac{T-\gamma}{\eta}\right)^{\beta}} \\
& \boldsymbol{F}(\boldsymbol{T})=\mathbf{1}-\boldsymbol{R}(\boldsymbol{T})
\end{aligned}
$$

\subsection{Mann Test}

In this research, initially all data are considered Weibull distribution. To prove whether the data is a Weibull distribution or not, a Mann test is performed. There are two hypotheses to do this test. H0: The failure time is Weibull and H1: The failure time is not Weibull. The Mann equation is below.

$$
\begin{gathered}
k_{1}=\left\lfloor\frac{r}{2}\right\rfloor, k_{2}=\left\lfloor\frac{r-1}{2}\right\rfloor \\
M_{i}=Z_{i+1}-Z_{i} \\
M=\frac{k_{1} \sum_{i k 1+1}^{r-1}\left[\left(\ln _{t+1}-\ln _{t}\right) / M_{i}\right]}{k_{2} \sum_{i=1}^{k 1}\left[\left(\ln _{t+1}-\ln _{t}\right) / M_{i}\right]} \\
Z_{i}=\ln \left[-\ln \left(1-\frac{i-0.5}{n+2.5}\right)\right]
\end{gathered}
$$

r: number of data failures

$\mathrm{n}$ : number of installed components

$\mathrm{k} 1$ : the amount of data before the median $\mathrm{k} 2$ : amount of data after median

2k1: denominator 2k2: numerator

If the value of $\boldsymbol{M}>$ Fcrit then H1 accepted, meaning the failure time is not Weibull.

Whereas if the value of $\boldsymbol{M}<\boldsymbol{F c r i t}$ then H0 accepted, it means that the failure time is Weibull. The value of Fcrit is determined by Ms. Excel using the F.INV.RT statistical function of alpha 0,05.

\subsection{Application Specifications}

Application specifications are determined by literature studies, problem formulations, observations of applications on the market and comparison with similar applications. The developed application feature is selected so that the application can accommodate all the specified application specifications. Application specifications that will be developed are as follows

a. The application can accommodate RCM / MSG-3 logic.

b. Can do data management in the database.

c. Can determine the probability of failure, risk of failure, effective maintenance tasks and maintenance intervals. 


\subsection{Application Flow}

The application will be developed based on the specifications that have been obtained and will be formulated in the application development chapter. The use of RCM/MSG will be the basis for developing applications that are connected with risk analysis.

a. Input Item Attribute. Enter the failed item attribute to be analyzed. Input item names, item numbers, item functions, item malfunctions, failure conditions, failure modes, failure effects and failure consequence

b. Input Equipment Life, Occurrence Threshold and Risk. They are will be entered by the user (user defined). Occurrence threshold will be used as a threshold on the consequences of safety and risk threshold as a threshold on the consequences of non-safety.

c. Occurrence. Occurrence is the probability of failure or called unreliability, $F(T)$. Determination of occurrence values is based on processing failure data using the calculation three parameters of Weibull Distribution. After finding the three parameters of Weibull, the occurrence value can be determined at the age of equipment life or maintenance interval

d. Risk. Risk is the multiplication between occurrence and severity. The results of this multiplication will be compared with the risk threshold that will be set by the user. The results of these comparisons will determine the item MSI's or Non-MSI's [2,4]. MSI is an item that if it fails has one or more of the following effects as follow:

(a) Not detected by the operating crew.

(b) Affect the safety and damage to the environment.

(c) Has a significant operational economic impact.

(d) Has a significant non-operational economic impact.

(e) Maintenance Tasks and Intervals.

Determination of maintenance tasks is carried out based on the effectiveness. The maintenance interval is the user define. For inspection task, maintenance interval determined half the P-F interval $[1,5]$. Determination of maintenance tasks is carried out based on the effectiveness of maintenance tasks.

In the failure consequences of safety, the occurrence at the interval is compared with the occurrence threshold. Effective if the occurrence is smaller than the occurrence threshold. For lubrication, the calculation of the occurrence value at the interval has a multiplier factor of 0.5 with consideration of the degradation that occurs when lubrication is carried out. In the failure consequences of non-safety, maintenance task is effective if they have to meet two criteria, namely the risk at the interval must be smaller than the risk threshold, and the comparison between decreasing severity and maintenance costs (PM Cost) in a certain period must be greater than the risk ratio that determined by the user. In this application the risk ratio value is recommended at five.

Task combination is carried out on consequences of safety for evident and hidden if an effective maintenance task is not found. Determination of this treatment task is the final choice by choosing two maintenance tasks that are considered the most effective, namely by looking at the value of the smallest occurrence. The second value of the occurrence will be multiplied so that a new occurrence value will be obtained for the task combination maintenance task.

\subsection{Calculation of Hidden Functional Failure}

In the calculation of multiple failure used the concept of system reliability block diagrams with parallel systems, which means that two or more components are arranged in parallel and all units must fail so that the system fails. If one or more units operate then the system will still operate (1 out of $n$ ), as presented on Figure 1. 


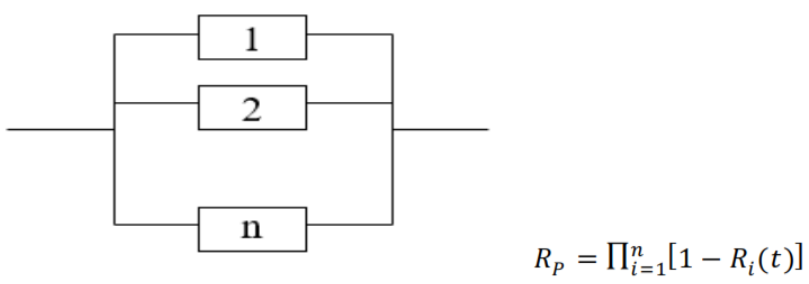

Figure 1. Parallel system block diagram.

To determine the reliability of multiple failure (RMF) is used the equation $\boldsymbol{R} \boldsymbol{M} \boldsymbol{F}=\prod \boldsymbol{n}[\mathbf{1}-\boldsymbol{R i}(\boldsymbol{t})]$ $\boldsymbol{i}=\mathbf{1}$ with $R i$ is the reliability of the $\mathrm{i}$ and $\mathrm{n}$ items is the number of items that fail [6]. By knowing the reliability value of multiple failure, the occurrence value of multiple failure OMF is OMF=1-RMF.

To determine the occurrence of multiple failure, doing evaluation of each component fails to determine the occurrence of each component and calculates the OMF. The OMF is compared to the occurrence threshold to determine the multiple failure of SI's or Non-MSI's. If multiple failure is MSI, it will determine the maintenance task for each failed component and calculate the OMF value after the maintenance task. In the consequence of safety, the OMF value after the maintenance task will be compared to the occurrence threshold. It says effective safety if the OMF value is smaller than the occurrence threshold.

In the consequences of non-safety, effective maintenance tasks are carried out if fulfilling two criteria, namely the results of risk multiple failure must be smaller than the risk threshold and the comparison value between severity reduction and maintenance costs in a certain period of time must be greater than the risk ratio. which is called the Efficient PM Cost where the value of the risk ratio is determined by the user. In this application the risk ratio is recommended at five.

\section{Results}

Database design is based on literature studies. After the data and information needed has been obtained, then data grouping is carried out. Grouped data into user data, item data, function data, failure data, and maintenance task data. Then a relation or relationship from one group of data to another data group is made. The design of the relationships of groups of data created in this application is shown in Figure 2.

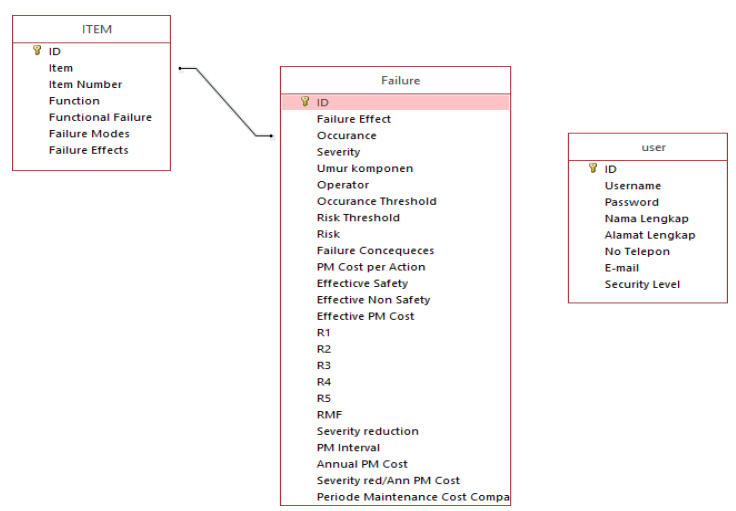

Figure 2. Relationship data.

Because the calculation of failure analysis is quite complex, Ms. Access can't do it so using Ms. Excel to accommodate these calculations. Integration between Ms. Access and Ms. Excel is done by giving the command button to Ms. Access.

The command button is set so can open Ms. Excel by creating code in Visual basic. Microsoft Access has the appearance of using a GUI (Graphical User Interface) that allows users to develop this application. Required settings regarding users who can use and manage various features in the 
application. This login feature uses "Username" for each user who has registered with "Password" for each existing username. There are differences in user levels, namely admin and user levels that are used to distinguish authority by users in the application. Admin-level users can access all features provided by the application while users do not.

The difference is that the admin can access the "Admin page" page which is a page for managing users who can use the application including adding, subtracting, determining user levels and determining the threshold value to be used in the analysis. In Figure 3 to Figure 10 will show the results of the application interface developed.

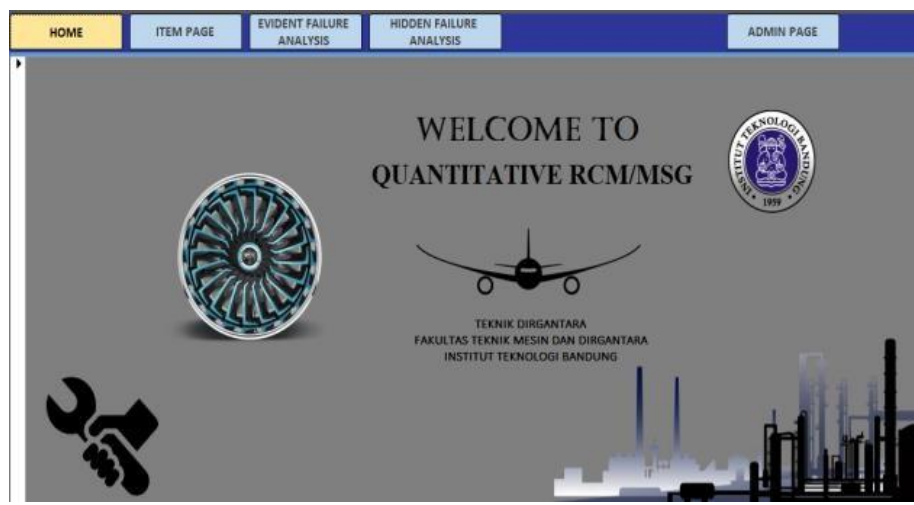

Figure 3. Home page display.

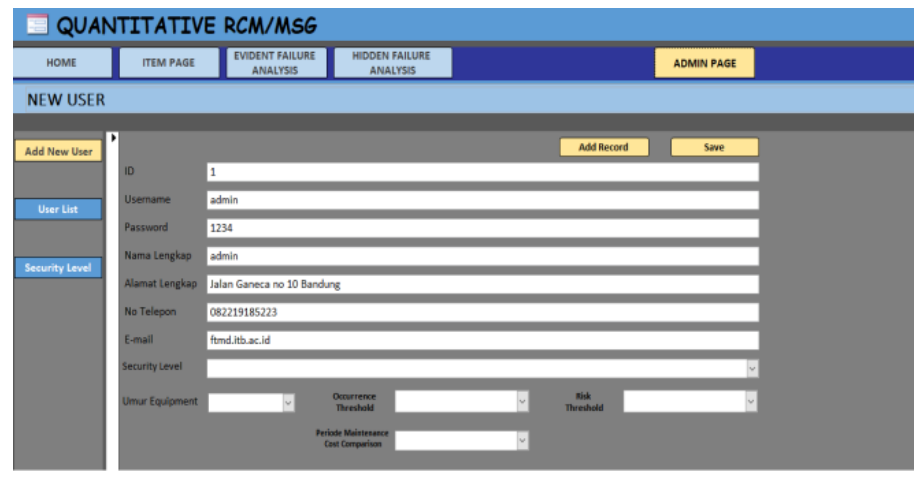

Figure 4. Admin page display.

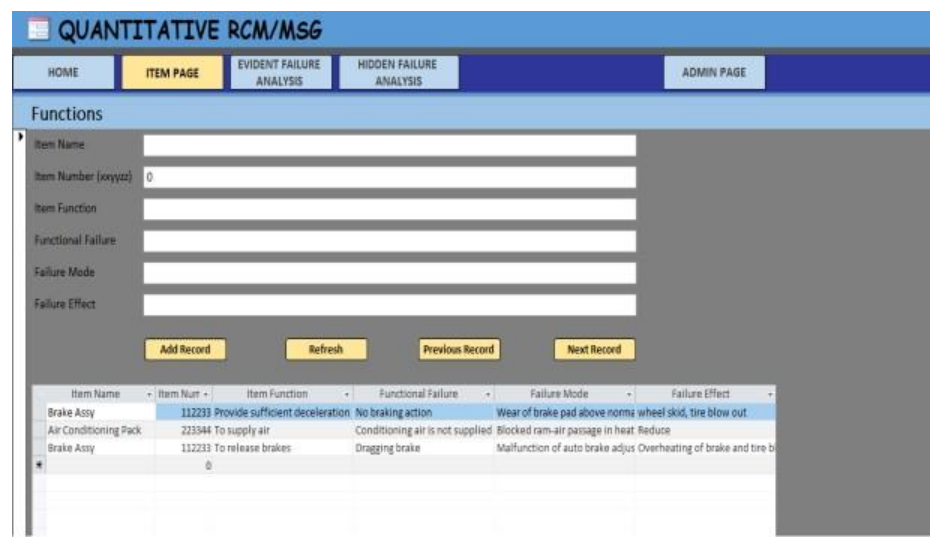

Figure 5. Item page display. 


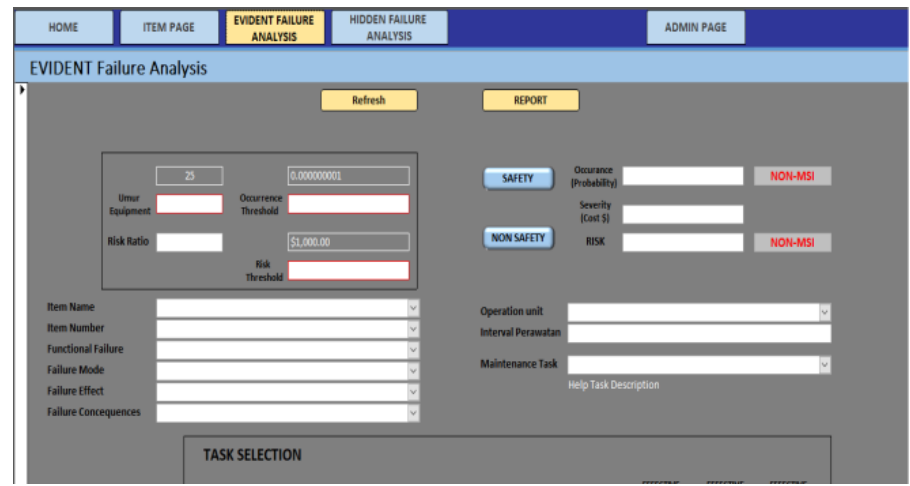

Figure 6. Evident failure analysis page display.

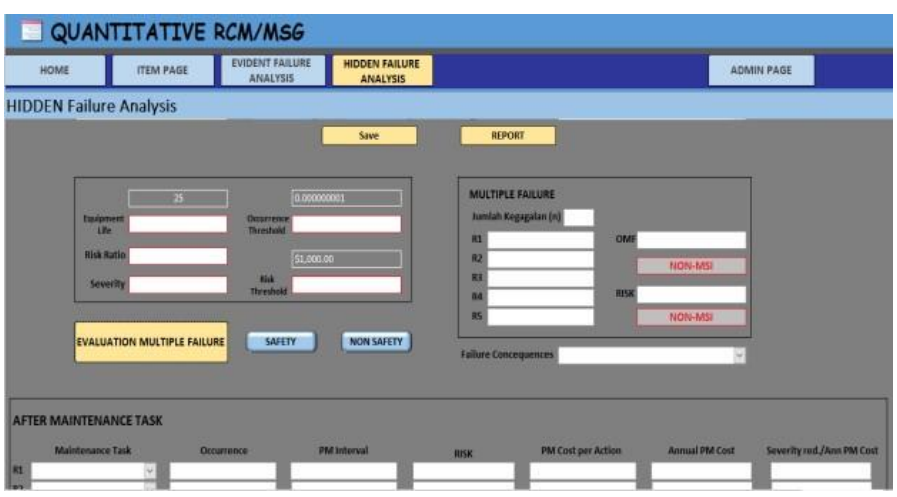

Figure 7. Hidden failure analysis page.

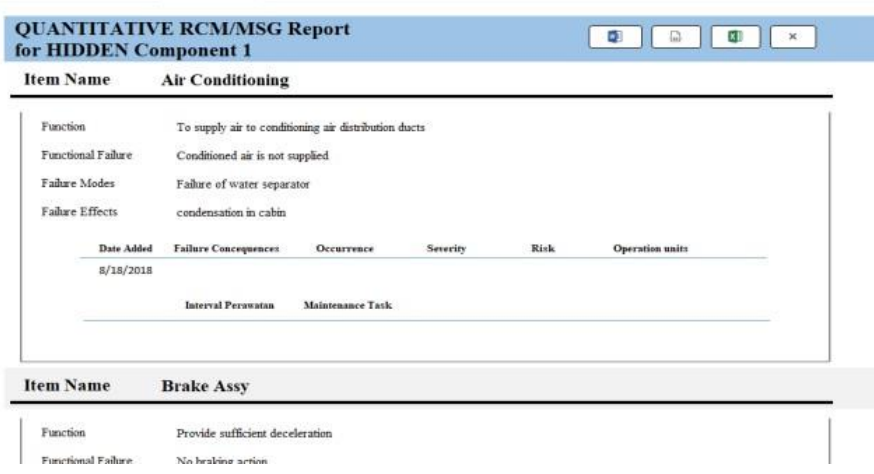

Figure 8. Report page.

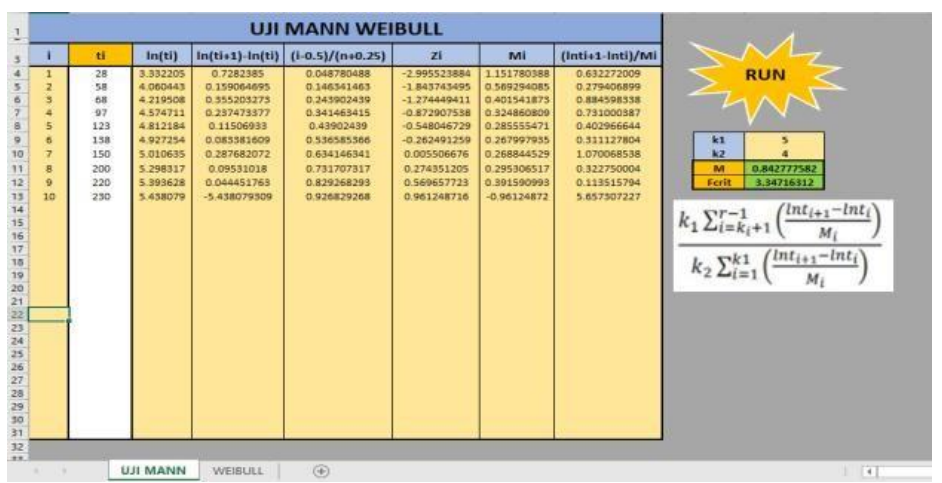

Figure 9. Mann test display page. 


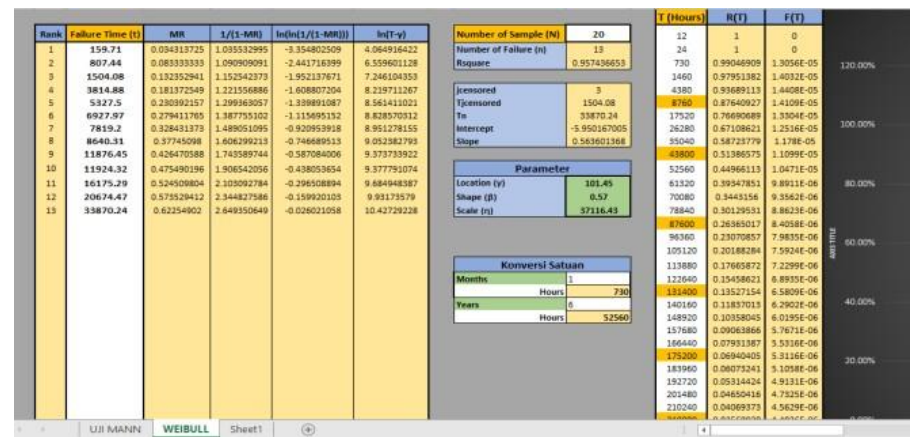

Figure 10. Failure analysis calculation page.

\section{Discussions}

\subsection{Application Validation}

Validation of Mann test on manual calculation application. Mann test on the application using the help of Visual Basic Macro on Ms. Excel. A dialog box will appear containing data according to the Weibull distribution or not. For example, an air conditioning has failure data in hours: 12, 120, $360,988,1080,3240,6480,7200,9900,10000,25500,45250$ in 18 samples. The application will get the results as shown in Figure 3. In manual calculations using the Man Test equation, the results shown in Table 1 will be obtained.

Table 1. Mann test manual calculation.

\begin{tabular}{|c|c|c|c|c|c|c|c|}
\hline $\mathbf{i}$ & ti & $\ln (\mathbf{t i})$ & $\ln (\mathbf{t} \mathbf{i}+\mathbf{1})-\ln (\mathbf{t i})$ & $(\mathrm{i}-0.5) /(\mathrm{n}+0.25)$ & $\mathbf{Z i}$ & Mi & $(\ln \mathrm{ti}+1-\ln \mathrm{ti}) / \mathrm{Mi}$ \\
\hline 1 & 12 & 2.484907 & 2.302585093 & 0.040816327 & -3.3498014 & 1.135220778 & 2.028314788 \\
\hline 2 & 120 & 4.787492 & 1.098612289 & 0.12244898 & -2.2145807 & 0.549852038 & 1.998014398 \\
\hline 3 & 360 & 5.886104 & 1.009578666 & 0.204081633 & -1.6647286 & 0.378291858 & 2.668782433 \\
\hline 4 & 988 & 6.895683 & 0.089033622 & 0.285714286 & -1.2864368 & 0.296401454 & 0.300381868 \\
\hline 5 & 1080 & 6.984716 & 1.098612289 & 0.367346939 & -0.9900353 & 0.24963737 & 4.400832648 \\
\hline 6 & 3240 & 8.083329 & 0.693147181 & 0.448979592 & -0.7403979 & 0.220711728 & 3.140509052 \\
\hline 7 & 6480 & 8.776476 & 0.105360516 & 0.530612245 & -0.5196862 & 0.202559576 & 0.520145813 \\
\hline 8 & 7200 & 8.881836 & 0.318453731 & 0.612244898 & -0.3171266 & 0.192004601 & 1.658573436 \\
\hline 9 & 9900 & 9.20029 & 0.010050336 & 0.693877551 & -0.1251220 & 0.187825227 & 0.053508978 \\
\hline 10 & 10000 & 9.21034 & 0.936093359 & 0.775510204 & 0.06270315 & 0.190274337 & 4.91970369 \\
\hline 11 & 25500 & 10.14643 & 0.573524218 & 0.857142857 & 0.25297749 & 0.20161827 & 2.844604404 \\
\hline 12 & 45250 & 10.71996 & -10.71995795 & 0.93877551 & 0.45459576 & -0.45459576 & 23.58129766 \\
\hline
\end{tabular}

The results of the $\mathrm{M}$ value are 0.825203 and Fcrit using statistical function F.INV.RT is 2.912976722. $\boldsymbol{M}<\boldsymbol{F}$ crit $(0.825203<2.912976722)$ then the failure time is Weibull. In Table 4.2 will be shown the results of the comparison between applications and manual calculations.

Table 2. Results comparison of Mann test on application and manual calculation

\begin{tabular}{c|c|c}
\hline & Application & Manual \\
\hline $\mathbf{k 1}$ & 6 & 6 \\
\hline $\mathbf{k 2}$ & 5 & 5 \\
\hline $\mathbf{M}$ & 0.8252032 & 0.825203 \\
\hline
\end{tabular}

Based on the results above it can be concluded that the results of the Mann test using applications and manuals are the same which means valid. 


\subsection{User Friendly Test}

The level of how easy the application is used by users is very useful in application development. This test is done by giving an assessment to the application user. This assessment uses the following criteria, as follows: $5=$ very easy; $4=$ easy; $3=$ doubtful; $2=$ rather difficult; and $1=$ difficult.

Respondents were eight ITB Aerospace Engineering students, including group A, five people who had mastered the Aircraft Maintenance Engineering Course material and group B, namely three students who had mastered or knew the material of Aircraft Maintenance Engineering Course. The following shows the results of the friendly tests in Table 3.

Table 3. User friendly test result

\begin{tabular}{c|c|c|c|c|c|c|c|c}
\hline & \multicolumn{7}{|c|}{ RESPONDED'S ASSESSMENT } \\
\hline \multirow{2}{*}{ Question } & \multicolumn{3}{|c|}{ A Group } & \multicolumn{3}{c}{ B Group } \\
\cline { 2 - 10 } & $\mathbf{1}$ & $\mathbf{2}$ & $\mathbf{3}$ & $\mathbf{4}$ & $\mathbf{5}$ & $\mathbf{6}$ & $\mathbf{7}$ & $\mathbf{8}$ \\
\hline $\mathbf{1}$ & 5 & 5 & 5 & 5 & 5 & 5 & 5 & 5 \\
\hline $\mathbf{2}$ & 5 & 5 & 5 & 4 & 4 & 4 & 5 & 5 \\
\hline $\mathbf{3}$ & 5 & 5 & 5 & 5 & 5 & 5 & 5 & 5 \\
\hline $\mathbf{4}$ & 5 & 5 & 3 & 5 & 5 & 5 & 4 & 5 \\
\hline $\mathbf{5}$ & 5 & 5 & 4 & 4 & 5 & 4 & 5 & 4 \\
\hline $\mathbf{6}$ & 5 & 5 & 3 & 4 & 5 & 4 & 5 & 5 \\
\hline $\mathbf{7}$ & 5 & 5 & 3 & 5 & 5 & 4 & 5 & 5 \\
\hline $\mathbf{8}$ & 4 & 4 & 5 & 5 & 4 & 5 & 5 & 5 \\
\hline $\mathbf{9}$ & 5 & 5 & 5 & 5 & 5 & 5 & 5 & 5 \\
\hline $\mathbf{1 0}$ & 4 & 4 & 4 & 5 & 5 & 4 & 5 & 5 \\
\hline $\mathbf{1 1}$ & 4 & 4 & 4 & 5 & 5 & 5 & 5 & 5 \\
\hline $\mathbf{1 2}$ & 4 & 4 & 5 & 5 & 5 & 4 & 3 & 5 \\
\hline $\mathbf{1 3}$ & 5 & 4 & 4 & 5 & 5 & 5 & 4 & 4 \\
\hline $\mathbf{1 4}$ & 5 & 5 & 5 & 5 & 5 & 5 & 5 & 5 \\
\hline AVARAGE & 4.714 & 4.643 & 4.286 & 4.786 & 4.857 & 4.571 & 4.714 & 4.857 \\
\hline
\end{tabular}

Figure 11 shows a result of the friendly-friendly test for all respondents are shown and a comparison of the level of user ease of each question can be seen. From the picture below and the table can be concluded that the application is easy to use.

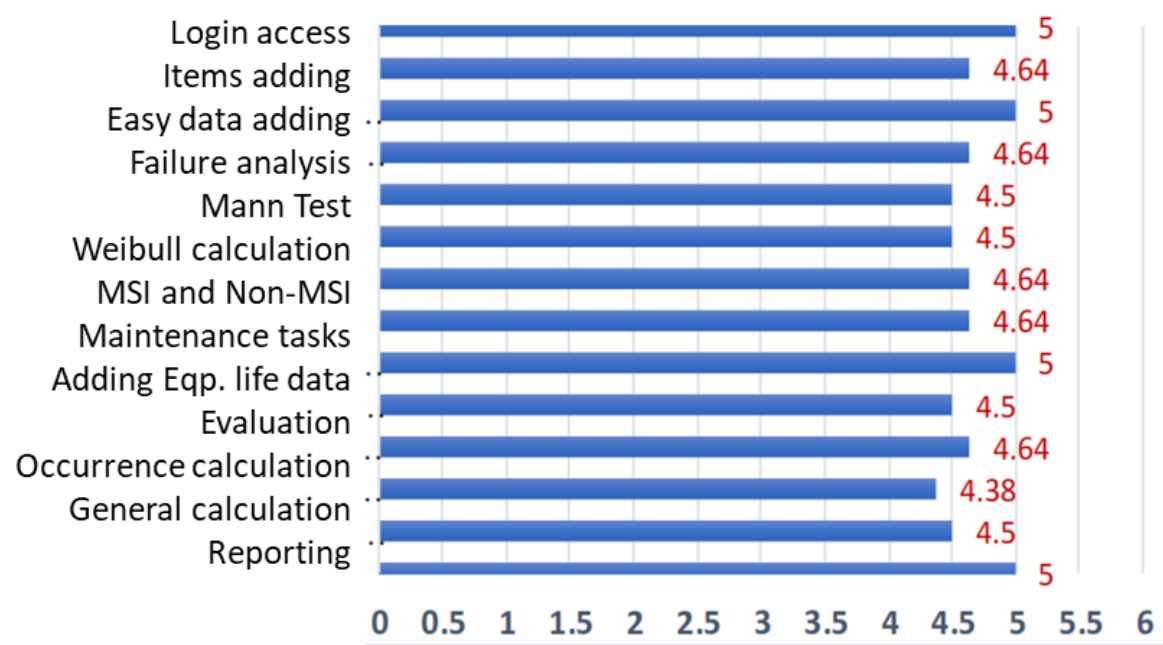

Figure 11. The results of the overall average user-friendly test 


\section{Conclusions}

The development of the RCM/MSG quantification application conducted in this study gives some insight that the specifications used to develop RCM / MSG applications using Microsoft Access shows that the application can accommodate RCM / MSG-3 logic, data management, determine the failure analysis, effective maintenance tasks and maintenance intervals. RCM/MSG-3 quantification application is called "QUANTITATIVE RCM/MSG" that can determine effective maintenance tasks and intervals for the category of evident and hidden failure effects by paying attention to their effects on safety and financial. This application was developed using Microsoft Access and Microsoft Excel for an easy to use and the value of risk can be a parameter of the selection of effective maintenance tasks in the RCM/MSG process. The effectiveness appraiser is based on a decrease in the risk of failure to an acceptable limit. If after a maintenance task is decrease value of the risk to the limit of the threshold, the maintenance task is effective.

\section{References}

1. E. Suwondo, Diktat Kuliah AE4170: Rekayasa Perawatan Udara, Bandung: Institut Teknologi, 2013.

2. ATA MSG-3, Operator/manufacturer; Scheduled Maintenance Development, Washington DC: Air Transport Association of America, Inc, 2002.

3. C. E. Ebeling, An Introduction to Reliability and Maintainability Engineering, Illinois: Waveland Press Inc, 2005.

4. K. Muralidhar and Z. S, A Simple Minimum-Bias Percentile Estimator of Location Parameter for Gamma, Weibull, and Loz Normal Distribution, Decisions Sciences, Vol. 23, No 4, 1992.

This is an open-access article distributed under the terms of the Creative Commons Attribution 4.0 International License, which permits unrestricted use, distribution, and reproduction in any medium provided the original work is properly cited. 